\title{
ADRENAL HORMONES AS INDEPENDENT PREDICTOR FACTORS OF MORTALITY DURING SEPSIS OR SEPTIC SHOCK
}

De Castro Fernández, Rocío ${ }^{1}$; Ruiz Ochoa, David ${ }^{2}$; Lamsfus Prieto, José Ángel${ }^{1}$; Lavín Gómez, Bernardo Alio ${ }^{3}$; García Unzueta, María Teresa ${ }^{3}$; Tejado Elviro, Iñigo2; Marcano Rodríguez, Gilberto ${ }^{1}$; Santibañez Margüello, Miguel ${ }^{4}$

${ }^{1}$ Anesthesiology and Reanimation. Sierrallana Hospital. Cantabria. Spain. ${ }^{2}$ Endocrinology and Nutrition. Sierrallana Hospital. Cantabria. Spain. ${ }^{3}$ Clinical Analysis. Marqués de Valdecilla Universitary Hospital. Cantabria. Spain. ${ }^{4}$ University of Cantabria-IDIVAL. Cantabria. Spain.

The activation of the hypothalamic-pituitary-adrenal axis is critical for adaptation to stress and serious illness. The imbalance between antiinflammatory activity of cortisol and proinflammatory activity of the adrenal androgens, dehydroepiandrosterone (DHEA) and dihydroepiandrosterone sulfate (DHEAS) can be an important predictor factor of mortality in sepsis.

\section{OBJECTIVES}

Determine the adrenal hormones ability to predict at 28 days mortality, in patients with severe sepsis or septic shock.

\section{MATERIAL AND METHODS}

Patients diagnosed of severe sepsis or septic shock who were admitted to critical unit of Sierrallana Hospital in Torrelavega (Spain). Cortisol, DHEA and DHEAS were determined in the first 24 hours of admission and mortality was recorded at 28 day. For each biomarker, Area Under Curve (AUC) and its $95 \%$ confidence interval (95\%CI) were estimated by using ROC curves. Levels of biomarkers were ordinal categorized in tertiles (T1, T2 and T3), and as association measure Odds Ratios (OR) with their $95 \% \mathrm{CI}$ adjusted for age, sex, SOFA and presence of severe sepsis or septic shock were estimated.

\section{RESULTS}

Table 1: Baseline characteristics

\begin{tabular}{|c|c|c|c|c|}
\hline & $\begin{array}{l}\text { TOTAL } \\
\mathbf{N}=72\end{array}$ & $\begin{array}{l}\text { SURVIVORS } \\
N=63\end{array}$ & $\begin{array}{l}\text { NO } \\
\text { SURVIVORS } \\
\mathbf{N}=9\end{array}$ & $\mathbf{p}$ \\
\hline $\begin{array}{l}\text { AGE } \\
\text { Median (IQR) }\end{array}$ & $67.2(58.4-76.3)$ & $\begin{array}{l}65.1(56.2- \\
75.3)\end{array}$ & $\begin{array}{l}80.39(69.6- \\
82.2)\end{array}$ & 0.004 \\
\hline $\begin{array}{l}\text { SEX } \\
\text { n (\%) }\end{array}$ & $\begin{array}{l}\text { M: } 43(59.7) \\
\text { W: } 29(40.3)\end{array}$ & $\begin{array}{l}\text { M: } 41 \text { (95) } \\
\text { W: } 22(76)\end{array}$ & $\begin{array}{l}M: 2(5) \\
W: 7(24)\end{array}$ & 0.025 \\
\hline $\begin{array}{l}\text { SEPTIC SHOCK } \\
\mathrm{n}(\%)\end{array}$ & $29(40,3)$ & $24(82.8)$ & $5(17.2)$ & 0.525 \\
\hline $\begin{array}{l}\text { APACHE II } \\
\text { Mean(SD) }\end{array}$ & $15.7(7.0)$ & $15.02(7.3)$ & $18.3(5.1)$ & 0.051 \\
\hline $\begin{array}{l}\text { SOFA } \\
\text { Mean(SD) }\end{array}$ & $6(3.5)$ & $5.7(3.5)$ & $7(3)$ & 0.276 \\
\hline $\begin{array}{l}\text { PCT } \\
\text { Median (IQR) }\end{array}$ & $6.8(3.0-41.4)$ & $6.0(2.8-43.0)$ & $\begin{array}{l}9.73(5.24- \\
40.4)\end{array}$ & 0.640 \\
\hline
\end{tabular}

Table 2: Linear trend, crude OR and adjusted OR for age, sex, SOFA scale or diagnosis of severe sepsis or septic shock

\begin{tabular}{|c|c|c|c|c|c|}
\hline & Tertiles & cOR & $95 \% \mathrm{Cl}$ & aOR & $95 \% \mathrm{Cl}$ \\
\hline \multirow{3}{*}{$\begin{array}{l}\text { TOTAL } \\
\text { CORTISOL } \\
(\mu \mathrm{g} / \mathrm{dL})\end{array}$} & $\leq 13.50$ & 1.00 & -- & 1.00 & -- \\
\hline & $13.60-21.80$ & $0.96^{*}$ & $0.12-7.40$ & $1.98^{*}$ & $0.19-21.22$ \\
\hline & $>21.80$ & $3.24^{* *}$ & $0.56-18.7$ & $4.98^{* *}$ & $0.59-41.90$ \\
\hline \multicolumn{2}{|l|}{ Linear trend } & \multicolumn{2}{|l|}{$p=0.16$} & \multicolumn{2}{|l|}{$p=0.12$} \\
\hline \multirow{3}{*}{$\begin{array}{l}\text { TOTAL } \\
\text { CORTISOL/ } \\
\text { DHEA } \\
\left(\mu \mathrm{g} \bullet \mathrm{dl}^{-1} / \mathrm{ng} \bullet\right. \\
\left.\mathrm{ml}^{-1}\right)\end{array}$} & $\leq 4.62$ & 1.00 & -- & 1.00 & -- \\
\hline & $4.63-7.70$ & $0.95^{*}$ & $0.06-16.2$ & $0.71^{*}$ & $0.03-17.20$ \\
\hline & $>7.70$ & $10.00^{* *}$ & $1.10-90.59$ & $9.43^{* *}$ & $0.62-143.27$ \\
\hline Linear trend & & $P=0.01$ & & $P=0.03$ & \\
\hline \multirow{3}{*}{$\begin{array}{l}\text { TOTAL } \\
\text { CORTISOL/ } \\
\text { DHEAS } \\
\text { (ng/ng) }\end{array}$} & $\leq 0.19$ & -- & -- & -- & -- \\
\hline & $0.20-0.59$ & 1.00 & -- & 1.00 & -- \\
\hline & $>0.59$ & $2.24^{* * *}$ & $0.48-10.3$ & $2.04^{* * *}$ & $0.34-12.22$ \\
\hline
\end{tabular}

"Cortile ( First tertile category without deaths)
Graphic 1: ROC curves and AUC

Total Cortisol AUC: $0.74 ; 95 \%$ CI (0.55-0.93)

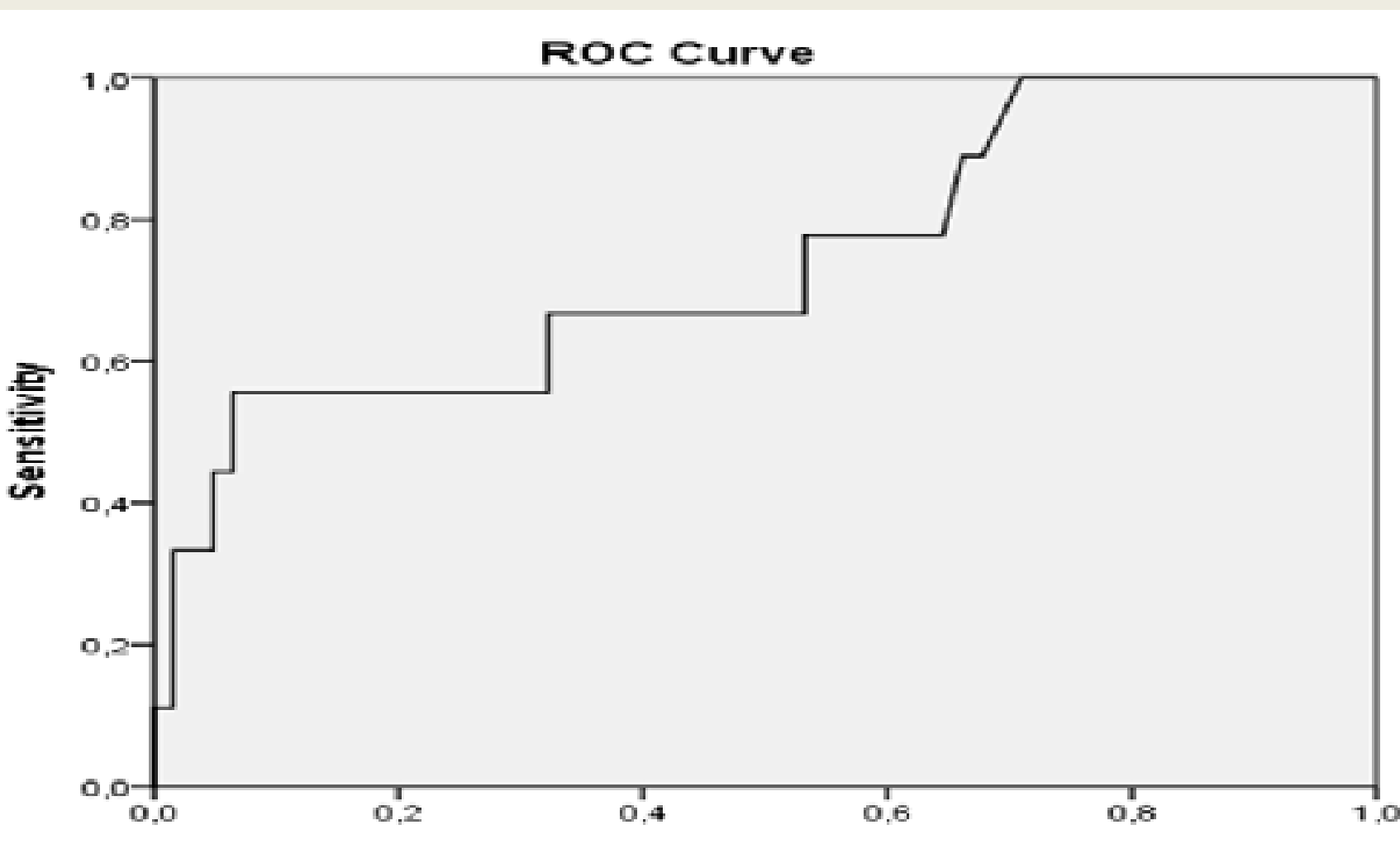

Cortisol/DHEA AUC: $0.74 ; 95 \%$ CI (0.55-0.92) Cortisol/DHEAS AUC: $0.79 ; 95 \%$ CI (0.65-0.94)
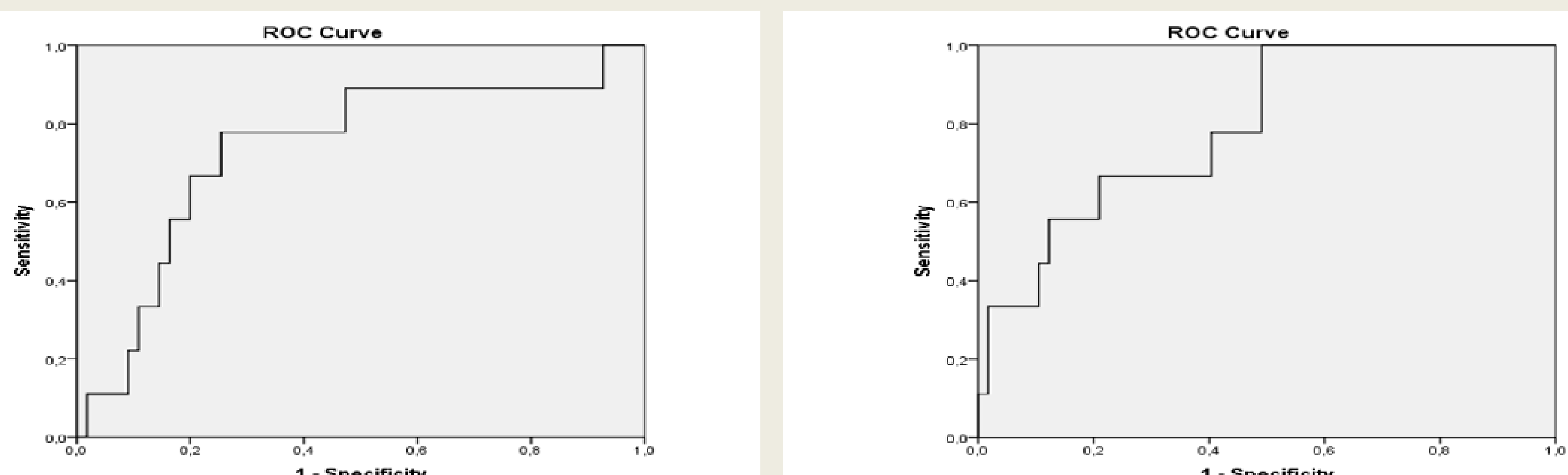

Table 3: AUC adjusted for albumin levels

\begin{tabular}{|c|c|c|c|c|c|c|}
\hline & AUC & $95 \%$ CI & $\begin{array}{l}\text { AUC } \\
(\text { Alb } \geq 2,5 \\
\mathrm{nmol} / \mathrm{I})\end{array}$ & $95 \%$ CI & $\begin{array}{l}\text { AUC } \\
(\text { Alb }<2,5 \\
\text { nmol/I) }\end{array}$ & $95 \% \mathrm{Cl}$ \\
\hline CORTISOL $(\mu \mathrm{g} / \mathrm{dl})$ & 0,74 & $0,55-0,93$ & 1 & $1-1$ & 0,71 & $0,52-0,91$ \\
\hline DHEA (ng/dl) & 0,55 & $0,34-0,76$ & 0,83 & $0,62-1$ & 0,38 & $0,15-0,61$ \\
\hline DHEAS $(\mu \mathrm{g} / \mathrm{dl})$ & 0,65 & $0,50-0,79$ & 0,46 & $0,16-1$ & 0,34 & $0,18-0,49$ \\
\hline $\begin{array}{l}\text { CORTISOL/DHEA } \\
\left(\mu \mathrm{g} \cdot \mathrm{dl}^{-1} / \mathrm{ng}^{\prime} \mathrm{ml}^{-1}\right)\end{array}$ & 0,74 & $0,55-0,92$ & 0,83 & $0,62-1$ & 0,75 & $0,54-0,95$ \\
\hline $\begin{array}{l}\text { CORTISOL/DHEAS } \\
(\mathrm{ng} / \mathrm{ng})\end{array}$ & 0,79 & $0,65-0,94$ & 0,91 & $0,74-1$ & 0,79 & $0,64-0,8$ \\
\hline
\end{tabular}

\section{CONCLUSIONS}

Markers of adrenal function are independent predictors of mortality in patients with severe sepsis or septic shock.

The predictive ability discriminated by the AUC of the ROC curve is higher for the ratios cortisol/DHEA and cortisol/DHEAs than for isolated values.

The predictive ability discriminated by the AUC of ROC curve is higher for normal levels of albumin than for low levels. 